\title{
The Relationship between Preference and Stare Duration on Bicycle
}

\author{
Jin-Han Tseng ${ }^{1}$, Ding-Bang Luh ${ }^{1}$, and Zhi-Hong Liang ${ }^{2}$ \\ ${ }^{1}$ National Cheng Kung University, Taiwan \\ ${ }^{2}$ Cycling \& Health Tech Industry R\&D Center, Taiwan \\ chinn60@yahoo.com, luhdb@mail.ncku.edu.tw, che@tbnet.org.tw
}

\begin{abstract}
Take bicycle industry as an example, the purpose of this research is to investigate the preference of consumer. With the using of eye-tracker, we expect to discover the relationship between preference of product form and the focus region of eye sight. With the help of eye-track technique and questionnaire research, this research aims to investigate the key design factor in bicycle design that influences customers' preference the most. According to the physical data of eye-tracker in this research, designers could make a better inference when doing redesign. Instead of starting all over again, changing the little part of an object may have a better chance to enhance overall preference. Hence, the time spent in production and redesign will be reduced. The result shows that there is a high correlation between preference and stare duration, in addition, frame of bicycle is the part that customer consider the most when they were asked to make decision. This result advice that if designers just want to change little part of bicycle but reach a higher return, they can focus on the frame especially the top tube and the down tube.
\end{abstract}

Keywords: bicycle, eye tracking, eye movement.

\section{Introduction}

Recently, the conscious of environment protection has risen, trend of LOHAS lifestyle, and widely construction of riding facility and the promoting of related policy, bicycle riding has been a an irresistible trend. Moreover, bicycle has become a representation of personal style. Besides, consumer-oriented marketing and the rising of KANSEI engineering cause the form of product become a key point to the success of a product. The forms of product make customer have different imagery and enhance their intention to purchase the product. (Seva, Duh, \& Helander, 2007) Therefore, how to develop a form that corresponds to consumers' ideal within a short period of time during design process is the problem that we want to investigate. When making purchase decision, customer will stare at the target product to see if the products fit their requirement. Hence, this research assumes that the part they look at longer is the part that they concern the most.

When looking at something people are interested in, they usually stare longer. But how do we know the relationship between staring time and preference. This study 
take bicycle industry as experiment material, and eye-track technique to detect the trace when participant watch the picture showed. The result showed that the time duration really has something to do with preference.

Purchase decision making is a complicated process, when customer are making their purchase decision, usually they will look around the target product, and further look at some particular part they are interested in, and then make up their mind. This study is interested in the relationship between preference and stare duration time, when making purchase decision, do customer stare longer at something they like, or they do not like? This research aims to figure out the relationship between stare time and preference, if we know the relationship between them, have better interpretation of how to refine the bicycle from redesign the most important part.

Hence, the research question of this research is: Do customer have different preference toward different part of bicycle? What are the reasons that make them have difference preference? And is the preference influence the focus region when they are making purchasing decision? We hope through using eye tracker to record the process when participants' are making decision. Furthermore, by analyze the watching duration; find out the focus point and their preference through post-eye track questionnaire.

\section{Eye Movement}

\subsection{Form Recognition}

The form of a product plays an important role on first impression that customer get, and further affect their purchase decision making. According to the research of Seva et al. (2007), design features are correlated to the perceived satisfaction of users expressed in 10 dimensions, namely luxuriousness, simplicity, attractiveness, colorfulness, texture, granularity, harmoniousness, salience, ruggedness, and overall satisfaction. In relative research of design cognitive, researcher proposed a hypothesis that "image with more preference has longer stare duration", and further investigate the effect of advertisement, the layout of website and the color cognition of infant (Pieters, Rosbergen, \& Wedel, 1999). A conclusion can be made that the stimulus people's receive from outside can really change the thought and may cause further influence, so is the form of product. Hence, if we can know the cognition process when consumers are making purchase decision, then some helpful design suggestion can be propose to the bicycle industry.

\subsection{Eye Track Technique and Related Application}

Traditional research method, such as questionnaire or interview, can only find general thought from participant. When participants are taking test, normally they will modify their answer and hide their original thought, so researchers cannot investigate the genuine result they expect to find. Hence, there are few methods that are used to detect if the answer researcher get from subjects are trustworthy enough by detect the physical evidence such as body temperature, heartbeat, and the last, eye-movement. 
This application aims to understand observers' emotion cognition. (Williams, Loughland, Green, Harris, \& Gordon, 2003)Eye gaze tracking is the process of making a computer aware of the gaze location of a participant (Liu, Ma, Zhang, \& Wang, 2010). Many researchers have applied eye movement in their researches to investigate cognitive process of participant on reading. Eye movement data provide a detailed reflection of cognitive information processing in many different kinds of displays such as the browsing process on website, cellphone, books.(Kevin et al., 1989) For example, Khushaba et al. (2013) investigated physiological decision processes while participants undertook a choice task designed to elicit preferences for a product by using eye tracker. Djamasbi, Siegel, and Tullis (2010) investigate the Y generation's cognition process while browsing website, and the reasons that catch their eyes.

\subsection{Measuring Eye Movement}

There are several method used to analyze eye-track data based on different technology, the application of eye-tracking technique has existed for over one hundred years. In early research, most of researchers have focused on readers' attention and eye movement, such as saccade and fix points, reading process (Hoffman, 1998). In general, there are three methods that usually used in eye track data analysis, including duration, trace and the focus region (Goldberg \& Helfman, 2010). In this study, we output the stare duration to make analysis, according to the definition, the experiment define the point that people stare longer than $20-40 \mathrm{mms}$ (thousandths of a second) as saccade, and the point longer than $200-250 \mathrm{mms}$ as fixation point (Russo 1978). Each point has its duration time, in the end we sum up all points in particular part, can get the time duration in different parts.

\section{Experiment}

\subsection{Participant Selection}

Due to the limitation, this study choose 36 convenient samples, which are composed by college or graduate students, half of female, half of male, aged form 18-24, and each of them has bicycle riding experiment for more than 5 years, as the participants of this experiment.

\subsection{Sample Collection}

This study used video based table-mounted eye tracker to record the trace of participants. As the goal is to find out the relationship and customers' preference, through analyzing the eye movement of participant toward 7 bicycle, including road bike, folding bike, mountain bike, which urban commuter ride commonly, and the questionnaire. This experiment set two main processes to approach the goal, first part is the main experiment which the eye-tracker will be used, and the other is questionnaire which intends to do the recheck of the eye-track data. 
The bicycle samples used in this experiment are selected from those released in 2012 from Giant Company. In order to make it more significant, the sample choose are more different with each other. Below is the list of sample. So there are 16 bicycles as below, CHIRON 2, IDIOM 1, MR 4F, FRESA, JOLLIOR, ARX, TCR COMPOSITE 3, DEFY 1, RAPID COMPOSITE 1, XTC COMPOSITE 2, ANTHEM X5, TRANCE X0, FAITH 1, AVAIL, AIMEZ COMPOSITE F, FRAIS. To narrow down the sample used in the main experiment and also make them look more separate, a pre-test were done before, participant are asked to rank over toward those 16 samples depend on their preference, and once they have down, the bicycle in the first place will be scored 16, and the last one will be scored 1. After all the participants done, this research choose the first three place and the last one in the rank of male's and female's, at the last, there were 7 bicycle which will be used into the next process, which are XTC COMPOSITE 2, RAPID COMPOSITE 1, CHIRON DS1, FAITH 1, AVAIL, AIMEZ COMPOSITE F, and ARX.

\subsection{Experiment Process}

The experiment was divided into two processes, first part, and also the main part is eye-tracker experiment, and the second is post-eye track questionnaire, which is used to compare with the first part.

In first part, participant will be asked to answer how much they like the bicycle shown on monitor. In order to imitate the circumstance that customer are doing bicycle purchasing evaluation, participants were asked to imagine that they are in the bicycle shop and want to buy a bicycle without the consideration about budget, under that situation they had to answer the questions: How much level do you want to buy this bicycle? (Liker-scale 1-7) And in the experiment, we make the sequence of pictures to be totally random, so that it can reduce the tiredness effectiveness on each participant. By asking this question, we can know that when customer are doing purchasing evaluation which part will they focus on and how do they make the evaluation.

In order to clear the interruption and noise between pictures, we set a black screen with a white cross in the middle between every screen, it will show for 3 seconds and then switch to the next slide (bicycle pictures) automatically, by doing this, the start point of every single screen, every participants' will restart from the middle.

The second part is the post-eye track questionnaire; this questionnaire aimed to recheck the data from eye-track experiment and also offer more information. In the questionnaire, participants were asked to answer the question about which parts they like the most, and the part they dislike the most, the answer were circled on the questionnaire. On the questionnaire, there are the selected 7 bicycle pictures, and below each picture listed some reasons to let them choose, for example, attractive, fashion, streamline etc. After participants all finish two experiments, we calculate how much circle each part have gained, and also the reasons; so that we can know the most like and dislike part, and those reasons. In this experiment, we expect to find out the emphasis area, stare duration, and by asking participant to highlight the part they feel "like" or "dislike", and the reasons, we can know the Factors that influence the preference. 


\section{Result}

By merging the entire export picture, we can see where the emphasis area that participants notice the most, like figure 1 . From the picture, we can notice that the frame of bicycle attract most of the attention.

Because the hypothesis of this study is will consumer stare longer on the part they like or what they don't like? In other words is that we want to find out the relationship between time duration and preference, hence, the regression statistic method are used in this research. According to the experiment mentioned before, we had the stare duration data of those 7 bicycles and the post-questionnaire provide the reasons of participants' most-like and most-dislike part, this research let these parts which have more participants circled on and their total time duration to do the regression statistic. In order to analyze the data systematically, we make all the bicycle have its coding map, so that we can categorize those circle that participant choose, there are 18 parts in total, and then we can calculate it and compare with the eye-tracking data.

In time duration, we sum up all the fixation points' time and gain the total duration time. The eye-track system used here will export the data chronologically, so that we can know if the focus region will change with time.

According to the post-eye track questionnaire, we have the part that participants' like and dislike the most, so the regression results are showed below. Table 1 is the statistics result, we have $\mathrm{R}$ square $=.055, \mathrm{~F}=.005$, which means they have correlation, instead, "like" and "time duration" does not reach the significant level, so there is not a relationship between them.

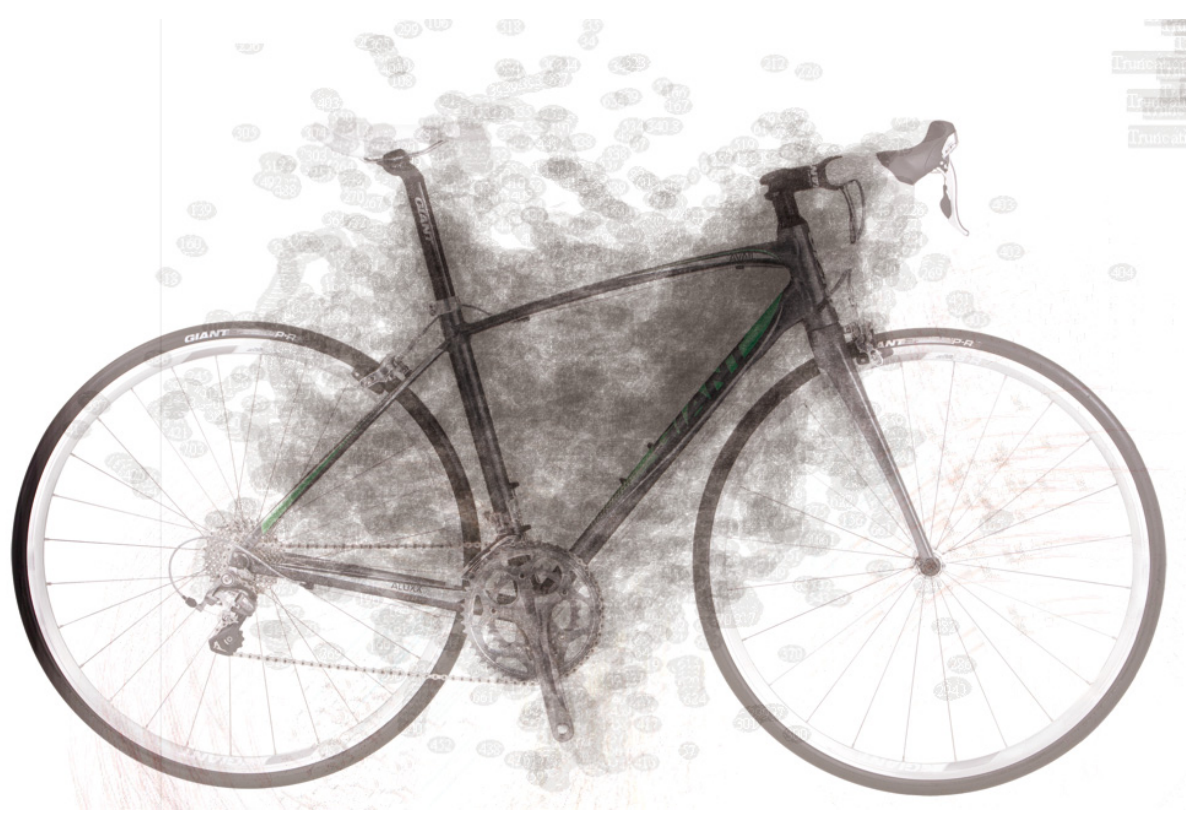

Fig. 1. Focus region 
From the table we notice that the part participant dislike have more strong relationship ( $\mathrm{t}=1.487$ ) to the time duration, therefore, we can infer that those part that is not good enough to fit their mind may influence their purchase willing. Moreover, those parts that most participants stare at are top tube and down tube, and they are also the parts customers like or dislike parts. This research also analyzes the watching procedure, we divided it into 3 parts, and each part has 4 seconds. It helps us to find out customers' most-emphasis part in different period. Furthermore, after merge all the result from 36 participants, we have the picture as shown below, it also explain which parts catch customer's attention.

Table 1. Dependent variables: time duration

\begin{tabular}{|l|c|c|c|c|}
\hline \multirow{2}{*}{ Model } & Adjusted $\mathrm{R}^{2}$ & Correlation const.. & $\mathrm{t}$ & Sig. \\
\hline like & -0.008 & .017 & 1.487 & .852 \\
dislike & 0.055 &. .215 & .125 & .005 \\
\hline
\end{tabular}

\section{Discussion}

From above analyze we can know that there do have some relationships between the stare time and preferences, in all, parts people dislike, usually they will stare at longer. According to the result, some points are discussed below.

Watching process: From eye-tracking data, we can know the watching procedure, usually they will start at top tube and down tube, we infer that maybe it is due to these part located in the middle of the see sight, so participant will easily notice it first, so it also influence them most. When we ask how much participants are willing to purchase that bicycle, at first (first four seconds), participants tend to stare at parts that most attract them, and most of the time they will look at that part, rest parts they just browse it. But at the end (last four seconds), they tend to see the part that they do not like. In all, they pay more attention on the part they dislike when making decision.

Emphasis area: basically, as the picture showed previously, they tend to look at the part they feel dislike, which are the part in the middle. As the time duration, which is compare with the post-eye track questionnaire, it points out that when people are making purchasing decision, they usually stare at the parts they like more than dislike.

Factors that influence preference: from the questionnaire, we can know that customer prefer bicycle form the feel stable, light, and swift, and also from the experiment, we can know that the width of the tube can have influence to the customers.

From above, here are some advices given here, first, since we can know when customer making decision, they tend to stare at the part they dislike, then when designer are doing design, they should focus on these parts to see how to modify it, then it may help get an evaluation on overall. Second, according to the result we can know that, customer usually care about the some important factors on the form of bicycle, which also offer some important design elements to future. If we can do the redesign part depend on the result, and do it again, it may have some important method that can be used in the future. 


\section{References}

1. Djamasbi, S., Siegel, M., Tullis, T.: Generation Y, web design, and eye tracking. International Journal of Human-Computer Studies 68(5), 307-323 (2010)

2. Goldberg, J.H., Helfman, J.I.: Visual scanpath representation. In: Paper presented at the Proceedings of the 2010 Symposium on Eye-Tracking Research \& Applications (2010)

3. Hoffman, J.E.: visual attention and eye movements (1998)

4. Kevin, F.J., Schmitt, N., Schechtman, L., Susan, H., Brian, M., Doherty, L.: Processing tracing methods: contributions, problems, and neglected research questions (1989)

5. Organizational Behavior and Human Performance, 43, 75-117

6. Khushaba, R.N., Wise, C., Kodagoda, S., Louviere, J., Kahn, B.E., Townsend, C.: Consumer neuroscience: Assessing the brain response to marketing stimuli using electroencephalogram (EEG) and eye tracking. Expert Systems with Applications 40(9), 3803-3812 (2013), http:/ / x. doi.org/10.1016/j .eswa.2012.12.095

7. Liu, R., Ma, S., Zhang, M., Wang, L.: Implementation and optimization of the eye gaze tracking system based on DM642. In: International Conference on Paper presented at the Intelligent Networks and Intelligent Systems (ICINIS), 3rd edn. (2010)

8. Pieters, R., Rosbergen, E., Wedel, M.: Visual attention to repeated print advertising: A test of scanpath theory. Journal of Marketing Research, 424-438 (1999)

9. Seva, R.R., Duh, H.B., Helander, M.G.: The marketing implications of affective product design. Appl. Ergon. 38(6), 723-731 (2007), doi:10.1016/j.apergo.2006.12.001

10. Williams, L.M., Loughland, C.M., Green, M.J., Harris, A.W., Gordon, E.: Emotion perception in schizophrenia: an eye movement study comparing the effectiveness of risperidone vs. haloperidol. Psychiatry research 120(1), 13-27 (2003) 